\title{
On the Validity of Diffusional Model in Determination of Electric Transport Parameters of Semiconductor Compound
}

\author{
A. DUSSAN ${ }^{a, *}$ AND F. MesA ${ }^{a, b}$ \\ ${ }^{a}$ Departamento de Fisica, Universidad Nacional de Colombia, Carrera 30, Calle 45, Bogota, Colombia \\ ${ }^{b}$ Departamento de Ciencias Básicas, Universidad Libre, Bogota, Colombia
}

\begin{abstract}
In this work we have studied the variable range hopping as a predominant electronic transport mechanism for semiconductor materials used as absorbent layer in photovoltaic devices. Dark conductivity measurements were carried out from 120 to $420 \mathrm{~K}$ in $\mathrm{Si}, \mathrm{Cu}_{3} \mathrm{BiS}_{3}, \mathrm{SnS}, \mathrm{Cu}_{2} \mathrm{ZnSnSe}_{4}$, and CuInGaSe 2 thin films. In the low-temperature range, variational range hopping was established for all samples. Using classical equations from the percolation theory and the diffusional model, the density of states near the Fermi level $\left(\mathrm{N}_{\mathrm{F}}\right)$, as well as the hopping parameters ( $W$ - activation energy and $R$ - hopping range) were calculated. A correlation between both models allowed us to evaluate the validity of the diffusional model in semiconductor compounds.
\end{abstract}

DOI: $10.12693 /$ APhysPolA.125.171

PACS: 72.80.Ey, 73.50.-h, 73.61.Ga, 73.50.Gr

\section{Introduction}

In recent years, technology in the development of photovoltaic devices has focused its interest in the application of ternary and quaternary semiconductor materials for disposal as absorber layer in solar cells [1-4]. Of these materials one can emphasize compounds such as $\mathrm{Cu}_{3} \mathrm{BiS}_{3}, \mathrm{CuInGaSe} \mathrm{CIGS}_{2}$, $\mathrm{SnS}$, and $\mathrm{Cu}_{2} \mathrm{ZnSnSe}_{4}$ (CZTSe), among others. Mono- and microcrystalline silicon is still being investigated to develop thin film solar cells and optoelectronic devices [5,6]. The studies related to electric transport have focused on understanding the mechanisms governing the transport and lead to the quality of material for application in photovoltaic devices [7-9].

In this work measurements of conductivity $(\sigma)$ as a function of temperature for compounds of $\mathrm{Cu}_{3} \mathrm{BiS}_{3}$, CIGS, SnS, and $\mathrm{Cu}_{2} \mathrm{ZnSnSe}_{4}$ were done. Measurements were carried out between 120 and $420 \mathrm{~K}$. It was observed that the conductivity measurements as a function of temperature are characterized by the Arrhenius type behavior for the region of $T>300 \mathrm{~K}$, leading to thermally activated carrier transport between the bands. For the low temperature region it was found that the mechanism governing the transport between variable range hopping (VRH) is the localized states near the Fermi level.

A detailed study on the parameters that characterize the VRH transport is presented: activation energy and range hopping via percolation theory. We report, comparatively, the values obtained through the diffusion model for these parameters along with the density of states near the Fermi level for each of the compounds.

*corresponding author; e-mail: adussanc@unal.edu.co
From the correlation between the two models there is demonstrated the validity of the diffusional model for obtaining the parameters that characterize the hopping behavior of semiconductor materials.

\section{Diffusional model}

Most of the studies carried out has reported that for semiconductor materials for the region of low temperatures (i.e. for $T<273 \mathrm{~K}$ ), the conductivity does not have Arrhenius behavior that indicates a conduction process via thermal activation $[8,10,11]$. Contrary to the above the percolation theory has been widely used to study and explain the possible mechanisms of transport of semiconductor materials.

According to the Mott equation in the case of 3D [12] (see Eq. (1)) the density of states is considered constant over a range of $k_{\mathrm{B}} T$ energy around the Fermi level

$$
\sigma=\sigma_{0}^{*} \exp \left(-\left(\frac{T_{0}}{T}\right)^{1 / 4}\right),
$$

where $T$ is the absolute temperature, and $T_{0}$ are constants depending on the material. Using percolation theory it is possible to find the values of the parameters $W$ and $R$, which characterize the hopping transport, however, due to the discrepancy in the expected range for these parameters, we developed in a previous work [7] a model, called diffusional model, which takes into account on the one hand, the relationship between the diffusion coefficient and the probability of hopping, and on the other hand, the Einstein relation between mobility and diffusion coefficient and conductivity. We have found a factor of dependence between hopping parameters obtained through percolation theory obtained from the diffusion model, which significantly improved the value corresponding to the activation energies of variable range hopping (see Table I). 
TABLE I

Equations for $R$ and $W$ parameters governing VRH transport. $A=(128 / 9) \pi$ and $B=(9 / 8) \pi$ $1 / 4, \alpha-$ localization length, $k_{\mathrm{B}}-$ Boltzmann constant, $T$ - temperature [7].

\begin{tabular}{c|c}
\hline \hline Percolation theory & Diffusional model \\
\hline$W=k_{\mathrm{B}}\left(T_{0} T^{3}\right)^{\frac{1}{4}}$ & $W=\frac{A}{C^{4}} k_{\mathrm{B}}\left(T_{0} T^{3}\right)^{1 / 4}$ \\
$R=\frac{3}{8} T_{0}^{1 / 4} T^{-1 / 4}\left(\frac{1}{\alpha}\right)$ & $R=\frac{B}{C} T_{0}^{1 / 4} T^{-1 / 4}\left(\frac{1}{\alpha}\right)$
\end{tabular}

\section{Experimental}

Electrical conductivity measurements in temperature dependence were carried out in samples of $\mathrm{Cu}_{3} \mathrm{BiS}_{3}$, $\mathrm{CuInGaSe} 2, \mathrm{SnS}$, and $\mathrm{Cu}_{2} \mathrm{ZnSnSe}_{4}$ semiconductor compounds. Measurements were made using the Van der Pauw method (according to ASTM F76) in a vacuum system, by varying the temperature in a range of 120 to $420 \mathrm{~K}$. Al contacts were deposited for samples $\mathrm{Cu}_{3} \mathrm{BiS}_{3}$ and $\mathrm{SnS}$, Mo for $\mathrm{Cu}_{2} \mathrm{ZnSnSe}_{4}$ and $\mathrm{CuInGaSe}_{2}$ compounds. The arrangement of the contacts was coplanar type. Deposition parameters of the sample can be checked from reports made with each of the materials $[7,8,13]$.

\section{Results and discussion}

Figure 1a shows the conductivity curves obtained for the samples of the compounds CIGS, $\mathrm{Cu}_{3} \mathrm{BiS}_{3}, \mathrm{SnS}$, and CZTSe. We have additionally included one of the measurements carried out in microcrystalline silicon (m-Si), given that this material is also one of the semiconductors used in the manufacture of photovoltaic devices. Figure 1b shows the $\sigma$ of CZTSe compound separately. For the latter case, the high values in the conductivity of the material are attributed to the presence of $\mathrm{Cu}$ in the preparation stage [14].

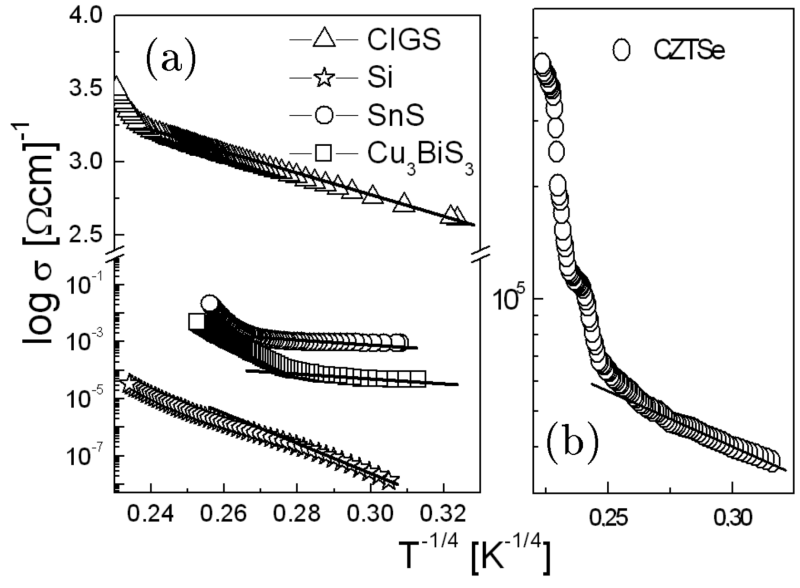

Fig. 1. Conductivity measurements as a function of $T^{-1 / 4}$ for (a) samples of CIGS, $\mathrm{SnS}$, and $\mathrm{Cu}_{3} \mathrm{BiS}_{3}$ semiconductor compounds. It includes a measurement with a sample of microcrystalline silicon (open circles). (b) $\mathrm{Cu}_{2} \mathrm{ZnSnSe}_{4}$ compound.

In Fig. 1 it is clearly visible that the conductivity curves as a function $T^{-1 / 4}$ exhibit behavior where low temperature can be adjusted with a linear function and this behavior demonstrates the transport of charge carriers via processes hopping between states present on the band-gap of the material. As established by Mott [12] the VRH is characterized by parameters that relate the energy gap between traps or activation hopping $(W)$ and the distance between jumps or hopping range $(R)$. Table II reports the values obtained by both the percolation theory and the diffusional model, VRH parameters for each of the samples.

TABLE II

Obtained values for the parameters $W$ and $R$ hopping in semiconductor samples from percolation theory (PT) and the diffusional model $(\mathrm{DM})$. The values for the density of states near the Fermi level $N\left(E_{\mathrm{F}}\right)$ were reported.

\begin{tabular}{c|c|c|c|c|c|c}
\hline \hline Sample & Location radio $[\AA]$ & $N\left(E_{\mathrm{F}}\right)\left[\mathrm{cm}^{-3} \mathrm{eV}^{-1}\right]$ & $R[\mathrm{PT}][\mathrm{cm}]$ & $W[\mathrm{PT}][\mathrm{eV}]$ & $R[\mathrm{DM}][\mathrm{cm}]$ & $W[\mathrm{DM}][\mathrm{eV}]$ \\
\hline $\mathrm{Si}$ & $10[7]$ & $2.139 \times 10^{16}$ & $1.46744 \times 10^{-6}$ & 0.36128 & $1.4695 \times 10^{-6}$ & 0.09628 \\
$\mathrm{Cu}_{3} \mathrm{BiS}_{3}$ & $257[15]$ & $3.269 \times 10^{17}$ & $3.83624 \times 10^{-6}$ & 0.05145 & $3.8415 \times 10^{-6}$ & 0.01293 \\
$\mathrm{CuInGaSe}_{2}$ & $257[15]$ & $1.579 \times 10^{17}$ & $4.60166 \times 10^{-6}$ & 0.06172 & $4.6080 \times 10^{-6}$ & 0.01551 \\
$\mathrm{SnS}$ & $257[15]$ & $8.139 \times 10^{19}$ & $9.65752 \times 10^{-7}$ & 0.01295 & $9.6709 \times 10^{-7}$ & 0.00326 \\
$\mathrm{Cu}_{2} \mathrm{ZnSnSe}$ & $257[15]$ & $3.403 \times 10^{18}$ & $1.25004 \times 10^{-7}$ & 0.02052 & $1.0332 \times 10^{-7}$ & 0.06359
\end{tabular}

Table II shows the values obtained for the range hopping both the diffusion model as from percolation theory are consistent with each other. However, hopping activation energy calculated from percolation theory is notoriously much higher than that obtained by the diffusion model. This was also observed in samples of microcrystalline silicon compensated with boron [7].

The values reported in Table II corresponding to $W$ from the diffusion model, confirm the initial assumption that the band-width around the Fermi level, which is responsible for driving the mechanism of VRH, is too narrow for the region of low temperatures. In accordance with the above it can be seen in Fig. 2 that the values of $R$ increase as the temperature decreases while the opposite occurs with hopping activation energy. This fact characterizes the mechanism of VRH, where carriers tend to perform jumps over great distances in order to find sites that remain energetically closer than its near neighbors. 


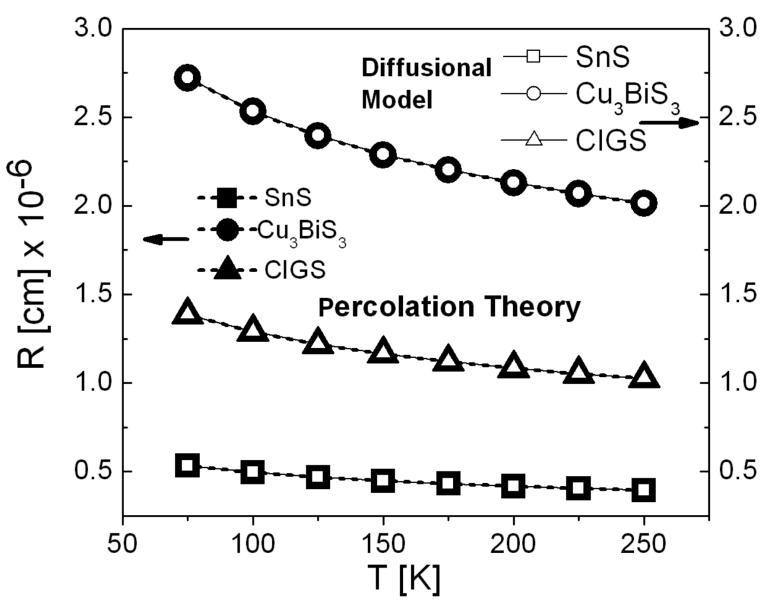

Fig. 2. Hopping range variation as a function of temperature of CIGS, SnS, and $\mathrm{Cu}_{3} \mathrm{BiS}_{3}$ semiconductor samples. Open symbols: diffusional model, closed symbols: percolation theory.

Table I shows that the calculated values for $N\left(E_{\mathrm{F}}\right)$ are consistent with those expected for materials that are used in the manufacture of photovoltaic devices $[7,16,17]$. Density of states acceptable for semiconductor materials must be established between a range of $10^{15}$ to $10^{20} \mathrm{~cm}^{3} \mathrm{eV}^{-1}$, with defect states in the gap that enable the transport of carriers. These states can be addressed and emptied into the energy dependence acquired by thermal processes.

\section{Conclusions}

Conductivity measurements were carried out on semiconductor samples of $\mathrm{Cu}_{3} \mathrm{BiS}_{3}$, CIGS, SnS, and CZTSe. It was shown that the transport mechanism that governs the low temperature region is the VRH. It was observed, in all cases, that a good agreement occurs between the values obtained for the parameter $R$ by both the percolation theory and the diffusion model. It was found that in diffusional model, the value of the hopping activation energy decreases substantially in accordance with the behavior expected for a VRH.

\section{Acknowledgments}

This work was supported by the Universidad National de Colombia, Quipu. 201010016486 Cod and Universidad Libre.

\section{References}

[1] P.-W. Li, W.-H. Zhou, Z.-L. Hou, S.-X. Wu, Mater. Lett. 78, 131 (2012).

[2] F. Mesa, A. Dussan, J. Sandino, H. Lichte, J. Nanopart. Res. 14, 1054 (2012).

[3] C.Z. Wang, C.J. Zhu, T.W. Zhang, J. Li, Vacuum 92, 7 (2013).

[4] E. Pineda, M.E. Nicho, P.K. Nair, H.L. Hu, Solar Energy 86, 1017 (2012).

[5] A. Dussan, H.P. Quiroz, J.G.A. Martinez, Solar En. Mater. Solar Cells 100, 53 (2012).

[6] S. Gall, C. Becker, E. Conrad, P. Dogan, F. Fenske, B. Gorka, K.Y. Lee, B. Rau, F. Ruske, B. Rech, H, Solar En. Mater. Solar Cells 93, 1004 (2009).

[7] A. Dussan, R.H. Buitrago, J. Appl. Phys. 97, 043711 (2005).

[8] F. Mesa, C. Calderon, G. Gordillo, Thin Solid Films 518, 1764 (2010).

[9] S.-Y. Lee, W.J. Lee, C.W. Nahm, J.M. Kim, S.J. Byun, T.H. Hwang, B.-K. Lee, Y.I. Jang, S.G. Lee, H.-M. Lee, B.W. Park, Curr. Appl. Phys. 13, 775 (2013).

[10] N. Parvathala Reddy, Rajeev Gupta, S.C. Agarwal, J. Non-Cryst. Solids 364, 69 (2013).

[11] C.-H. Lin, G.Y. Wu, Thin Solid Films 397, 280 (2001).

[12] N.F. Mott, Philos. Mag. 19, 333 (1969).

[13] A. Dussan, F. Mesa, M. Botero, G. Gordillo, J. Phys., Conf. Series 167, 012018 (2009).

[14] M.-L. Liu, F.-Q. Huang, L.-D. Chen, I-W. Chen, Appl. Phys. Lett. 94, 202103 (2009).

[15] M. Thamilselvan, K. Premnazeer, D. Mangalaraj, Sa.K. Narayandass, Physica B 337, 404 (2003).

[16] M.H. Lee, C.W. Tai, J.J. Huang, Solid-State Electron. 80, 72 (2013).

[17] S.S. Babkair, A.A. Ansari, N.M. Al-Twarqi Depa, Mater. Chem. Phys. 127, 296 (2011). 